\title{
Reinhard T, Larkin F (eds), Corneal Disease 2013, ISBN: 978-3-642-28746-6, Springer
}

\author{
Herbert E. Kaufman
}

Received: 2 July 2014 / Accepted: 8 July 2014 / Published online: 20 July 2014

(C) Springer-Verlag Berlin Heidelberg 2014

This is a multiauthor book with each chapter contributed by a different group. It focuses on recent developments, primarily involving the anterior segment and corneal disease in particular. There are seven chapters.

The first chapter, "The diagnosis and therapy of microbial keratitis", is a detailed account of the disease, especially as it occurs after LASIK. Although an excellent summary, it neglects to point out that mycobacteria are environmental pathogens, unlike most infecting organisms. They occur in water, drains, surfaces, etc. Recent improvements in operating room sanitation and instrumentation handling have drastically reduced the incidence of mycobacterial disease.

The second chapter, "New developments in antibacterial chemotherapy for bacterial keratitis", emphasizes the importance of culturing and identifying the specific causative organisms and using the optimal antibiotic for treating each infection. It discusses a number of antibiotics which are not yet available for general ophthalmic use.

The third chapter, "Heredity of keratoconus", is a detailed explanation of the techniques and present findings of possible genetic links to keratoconus. It does not discuss therapy.

The fourth chapter, "Advance corneal imaging", is an excellent discussion of in vivo confocal microscopy with detailed photographs and explanations of the value of this technique. It also discusses in detail, with very good illustrations, the use of computer tomography for anterior segment disease.
The fifth chapter, "Anti-angiogenic treatment options in the cornea", emphasizes the importance of lymphatic channels as well as blood vessels in causing transplant rejection and is a major contribution to the understanding of immunological rejection not only caused by vascular channels but by lymphatics. It discusses and details the use and potential of established and novel anti-angiogenic factors in improving corneal transplant results.

The sixth chapter is "The storage of donor cornea for penetrating the lamellar transplantation". These sections on the storage of tissue for endothelial keratoplasty are especially valuable and novel.

The seventh chapter, "Infant keratoplasty", is enormously valuable because of the tremendous experience the authors bring to this difficult and uncommon area of corneal transplantation. It seems balanced and especially useful for any clinician confronted with the necessity to do transplants in children. It provides an excellent estimate of the risks and potential visual benefits for transplantation in this age group.

In summary, this is a very good exposition of anterior segment subjects not generally considered in present textbooks, with knowledge often not easily available elsewhere. Naturally some chapters are better than others, but it is small, easy to read and valuable.
H. E. Kaufman $(\bowtie)$

Department of Ophthalmology, Louisiana State University, 2020

Gravier Street, New Orleans 70112, USA

e-mail: hkaufm@gmail.com 